\title{
Successful radiofrequency ablation of long-standing persistent atrial fibrillation in a patient with esophageal achalasia
}

\author{
Andrea Avella ${ }^{1 *}$, Piergiuseppe De Girolamo ${ }^{1}$, Francesco Laurenzi ${ }^{1}$, Augusto Pappalardo ${ }^{1}$, \\ Vitaliano Buffa ${ }^{2}$ \\ ${ }^{1}$ Cardiology Division and Cardiac Arrhythmia Unit, St. Camillo-Forlanini Hospital, Rome, Italy \\ ${ }^{2}$ Cardiovascular Imaging Unit, St. Camillo-Forlanini Hospital, Rome, Italy \\ Email: *andrea.avella@tin.it
}

Received 29 June 2012; revised 9 August 2012; accepted 20 August 2012

\begin{abstract}
A 54-year-old man was referred for ablation of symptomatic drug-refractory long-lasting persistent atrial fibrillation. His past medical history was negative for cardiac disease but included a diagnosis of esophageal achalasia. The patient underwent an ablation procedure, guided by barium esophagram, including isolation of the pulmonary veins, ablation of complex fractionated left atrial electrograms recorded on the septal wall and, finally, linear ablation of the cavo-tricuspid isthmus. The ablation procedure was performed with multielectrode ablation catheters using duty-cycled bipolar/unipolar radiofrequency energy. During 6 months of follow-up no recurrences of atrial fibrillation were documented. The reported case demonstrates how an ablation procedure for long-standing persistent AF may be safely performed even in a patient presenting with a achalasia, outlining the mega-esophagus position with a simple barium paste.
\end{abstract}

Keywords: Atrial Fibrillation; Ablation; Esophageal Achalasia

\section{INTRODUCTION}

At the present time, a number of different approaches are being employed to avoid esophagus-related complications during atrial fibrillation (AF) ablation procedures [1-6]. Barium paste, outlining the esophageal position in real time for the duration of the procedure, may provide an effective approach to minimize the potential risk of thermal injury when delivering radiofrequency (RF) energy at the posterior wall of the left atrium (LA), in close proximity to the esophagus [7].

In the case we here report barium esophagram was

"Corresponding author. successfully used to perform safely RF ablation of longstanding persistent $\mathrm{AF}$ in a patient with a megaesophagus due to achalasia, a rare neurodegenerative disease resulting in abnormal esophageal motility and impaired relaxation of lower esophageal sphincter [8].

\section{CASE PRESENTATION}

A 54-year-old man with symptomatic drug-refractory longstanding persistent AF was referred for catheter ablation after two failed electrical cardioversion. His past medical history was negative for structural heart disease but included a diagnosis of esophageal achalasia established 3 years earlier.

On admission, cardiac and chest computed tomography (CT) documented four distinct pulmonary veins (PVs) connecting with a LA, being partially compressed by an evident mega-esophagus (Figure 1 panels $A$ and B) with accumulation of undigested food and severe narrowing of its distal segment.

After outlining the mega-esophagus position with a small barium paste, swallowed by the patient prior to conscious sedation, a 3-step ablation procedure was performed with multielectrode ablation catheters using dutycycled bipolar/unipolar RF energy, (Medtronic - Ablation Frontiers, Carlsbad, CA, USA). Initially, electrical isolation of the four PVs was performed with a decapolar, circular, over-the-wire mapping and ablation catheter (Figure 1 panels $\mathbf{C}$ and D). Subsequently, an additional LA substrate modification was obtained targeting complex fractionated electrograms along LA septal wall (Figure 1 panel E) with a different multiarray ablation catheter. Finally, after restoration of the sinus rhythm by external electrical cardioversion, a linear ablation of the cavo-tricuspid isthmus was performed delivering RF energy with a specific hexapolar linear ablation catheter, until a bidirectional isthmus block was obtained (Figure 1 panel F). 

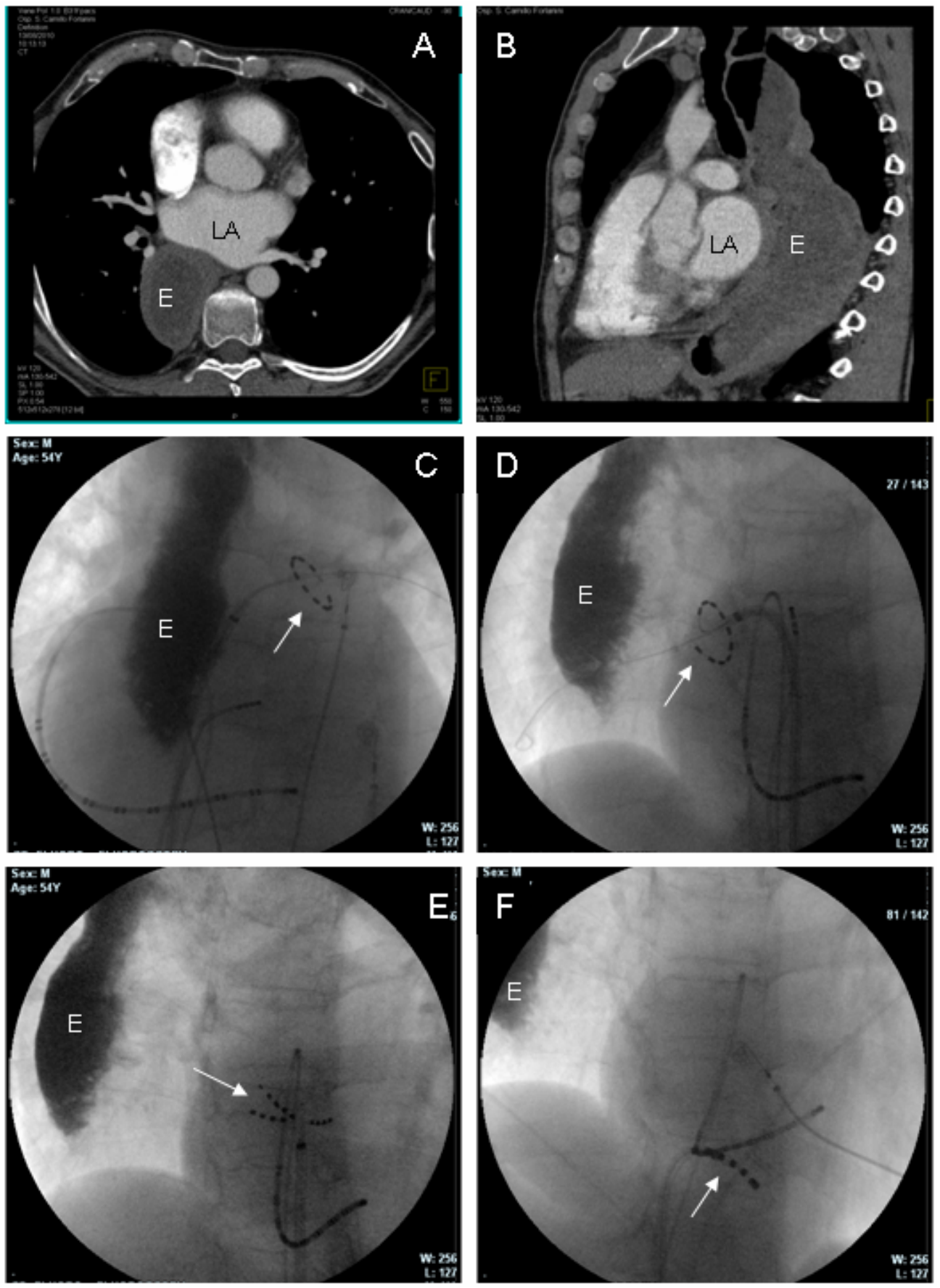

Figure 1. Panels A and B: Computed tomography scan showing a severely dilated and tortuous esophagus (E) compressing the left atrium (LA) posteriorly and almost completely filled with food debris (Panel A: axial view; Panel B: sagittal view). Panels C and D: fluoroscopy views showing PVAC $^{\mathrm{TM}}$ catheter (white arrow) while performing isolation of left superior (panel C-LAO view) and right inferior (panel D-RAO view) pulmonary veins. Also shown are a 4-polar catheter at the His bundle region and a 20-polar catheter mapping right atrium and coronary sinus. $\mathrm{E}=$ barium esophagram. Panel E: fluoroscopy RAO view showing MASC ${ }^{\mathrm{TM}}$ catheter (white arrow) used to perform defragmentation of the left atrial septal wall. Panel F: fluoroscopy RAO view showing TVAC $^{\mathrm{TM}}$ catheter (white arrow) used to perform linear ablation of the cavo-tricuspid isthmus.

While in the pre-ablation CT scan the mega-esophagus appeared directly adjacent to the posterior LA, during ablation fluoroscopy documented a significant separation between its lumen and the LA border, as a result of the 
spontaneous displacement of the esophagus. Accordingly, $\mathrm{RF}$ energy was safely delivered at an adequate distance from the esophagus and the ablation procedure was successfully performed with no complications. Two days after ablation the patient was discharged on therapy with warfarin and with a 3-month prescription of amiodarone. During 6 months of follow-up no recurrences of AF as well as no esophagus-related complications were documented.

\section{DISCUSSION}

The close proximity of the esophagus to the posterior LA has increasingly been emphasized in the last years to explain the rare but devasting cases of atrioesophageal fistula related to AF ablation procedures [9]. Many of the various approaches, currently used to minimize the potential risk of heat injury to the esophagus, aim at the identification of the esophageal position relative to the ablation catheter while delivering RF energy [1,2,4-6].

Barium paste, monitoring in real time the spontaneous movements of the esophagus relative to the posterior LA [7], may overcome the limitations of the static esophageal imaging obtained by pre-ablation CT scan and provide a simple and effective approach to avoid devasting complications when delivering RF energy at sites in the posterior LA.

Considering the intimate relationship of the esophagus with the LA, it is conceivable that the risk of thermal injury, during AF ablation, may result significantly increased in patients presenting a dilated esophagus compressing the posterior LA. The reported case demonstrates that even in a patient with a mega-esophagus due to achalasia, an ablation procedure of long-standing persistent AF may be safely performed, after obtaining real time monitoring of the mega-esophagus position with barium paste.

\section{CONCLUSION}

Barium esophagram seems to provide an accurate anatomical guidance to minimize the potential risk of thermal injury, during AF ablation procedures, even in patients presenting with mega-esophagus.

\section{ACKNOWLEDGEMENTS}

The authors thank biomedical engineer Simona Benedetti (Medtronic,
Italy) for her expert technical support.

\section{REFERENCES}

[1] Lemola, K., Sneider, M., Desjardins, B., et al. (2004) Computed tomographic analysis of the anatomy of the left atrium and the esophagus: Implications for left atrial catheter ablation. Circulation, 110, 3655-3660. http://dx.doi.org/10.1161/01.CIR.0000149714.31471.FD

[2] Kottkamp, H., Piorkowski, C., Tanner, H., et al. (2005) Topographic variability of the esophageal left atrial relation influencing ablation lines in patients with atrial fibrillation. Journal of Cardiovascular Electrophysiology, 16, 146-150. http://dx.doi.org/10.1046/j.1540-8167.2005.40604.x

[3] Redfearn, D.P., Trim, G.M., Skanes, A.C., et al. (2005) Esophageal temperature monitoring during radiofrequency ablation of atrial fibrillation. Journal of Cardiovascular Electrophysiology, 16, 589-593. http://dx.doi.org/10.1111/j.1540-8167.2005.40825.x

[4] Cummings, J.E., Schweikert, R.A., Saliba, W.I., et al. (2005) Assessment of temperature, proximity, and course of the esophagus during radiofrequency ablation within the left atrium. Circulation, 112, 459-464.

http://dx.doi.org/10.1161/CIRCULATIONAHA.104.5096 $\underline{12}$

[5] Ren, J.F., Marchlinski, F.E. and Callans, D.J. (2006) Real time intracardiac echocardiographic imaging of the posterior left atrial wall contiguous to anterior wall of the esophagus. Journal of the American College of Cardiology, 48, 594-595. http://dx.doi.org/10.1016/j.jacc.2006.05.019

[6] Ren, J.F., Lin, D., Marchlinski, F.E., et al. (2006) Esophageal imaging and strategies for avoiding injury during left atrial ablation for atrial fibrillation. Heart Rhythm, 3, 1156-1161.

http://dx.doi.org/10.1016/j.hrthm.2006.06.006

[7] Good, E., Oral, H., Lemola, K., et al. (2005) Movement of the esophagus during left atrial catheter ablation for atrial fibrillation. Journal of the American College of Cardiology, 46, 2107-2110. http://dx.doi.org/10.1016/j.jacc.2005.08.042

[8] Richards, W.O., Torquati, A. and Lutfi, R. (2005) The current treatment of achalasia. Advances in Surgery, 39, 285-314.

[9] Ghia, K.K., Chugh, A., Good, E., et al. (2009) A nationwide survey on the prevalence of atrioesophageal fistula after left atrial radiofrequency catheter ablation. Journal of Interventional Cardiac Electrophysiology, 24, 33-36. http://dx.doi.org/10.1007/s10840-008-9307-1 